\title{
CORRECTION
}

\section{Correction to: Thermal analysis and kinetic modeling of pulverized coal combustion accompanied with coke breeze}

\author{
Peng Han ${ }^{1} \cdot$ Wen-long Zhan ${ }^{1} \cdot$ Hao-bin $\mathrm{Zhu}^{1} \cdot$ Lei $\mathrm{Gao}^{2} \cdot{\text { Ying-chang } \mathrm{Yu}^{3} \cdot \mathrm{Zhi}^{-j u n ~} \mathrm{He}^{1} \cdot \text { Jun-hong Zhang }}^{1} \cdot$ \\ Qing-hai Pang'
}

Published online: 30 June 2021

(C) China Iron and Steel Research Institute Group 2021

Correction to: J. Iron Steel Res. Int. (2021) 28:809-817

The original article has been corrected.

$$
\text { https://doi.org/10.1007/s42243-021-00590-6 }
$$

The original version of this article unfortunately contained a mistake. Table 3 was incorrect, the corrected table is given below.

Table 3 Combustion characteristic parameters of single fuel

\begin{tabular}{|c|c|c|c|c|c|c|}
\hline Sample No. & $T_{\mathrm{i}} / \mathrm{K}$ & $T_{\mathrm{f}} / \mathrm{K}$ & $W_{\max } /\left(\% \min ^{-1}\right)$ & $W_{\text {mean }} /\left(\% \min ^{-1}\right)$ & $C /\left(\% \min ^{-1} \mathrm{~K}^{-2}\right)$ & $S /\left(\%^{2} \min ^{-2} \mathrm{~K}^{-3}\right)$ \\
\hline TA & 753 & 1059 & 14.2 & 9.7 & $2.50 \times 10^{-5}$ & $2.29 \times 10^{-7}$ \\
\hline HB & 645 & 942 & 15.3 & 10.8 & $3.68 \times 10^{-5}$ & $4.22 \times 10^{-7}$ \\
\hline $\mathrm{CB}$ & 835 & 1110 & 7.5 & 3.7 & $1.08 \times 10^{-5}$ & $3.61 \times 10^{-8}$ \\
\hline
\end{tabular}

The original article can be found online at https://

doi.org/10.1007/s42243-021-00590-6.

Wen-long Zhan

zhanwenlong288@163.com

$\triangle$ Lei Gao

glkust2013@hotmail.com

1 School of Materials and Metallurgy, University of Science and Technology Liaoning, Anshan 114051, Liaoning, China

2 Key Laboratory of Green-Chemistry Materials in University of Yunnan Province, Yunnan Minzu University, Kunming 650504, Yunnan, China

3 Ansteel Engineering Technology Corporation Limited, Anshan 114021, Liaoning, China 